\title{
A wayfinding aid to increase navigator independence
}

\author{
Wilfred Waters and Stephan Winter \\ Department of Infrastructure Engineering, University of Melbourne, Victoria 3010, Australia
}

Received: January 23, 2011; returned: April 24, 2011; revised: June 15, 2011; accepted: July 27, 2011.

\begin{abstract}
Wayfinding aids are of great benefit because users do not have to rely on their learned geographic knowledge or orientation skills alone for successful navigation. Additionally, cognitive resources usually captured by this activity can be spent elsewhere. A challenge, however, remains for wayfinding aid developers. Due to the automation of wayfinding aids, navigator independence may be decreasing via the use of these aids. In order to address this, wayfinding aids might be improved additionally to perform a training role. Since the most versatile wayfinders appear to deploy a dual strategy for geographic orientation, it is proposed that wayfinding aids be improved to foster such an approach. This paper presents the results of an experimental study testing a portion of the suggested enhancement.
\end{abstract}

Keywords: wayfinding aids, wayfinding strategy, navigation, sense of direction, geographic orientation, dual orientation strategy, route instructions, landmarks, piloting

\section{Introduction}

With mobile information and communication technology maturing, wayfinding aids (WAs) such as car navigation systems, personal navigation devices (PNDs), and web-based route planners are becoming increasingly popular. Much prior work on WAs has been aimed at increasing their usability (see, e.g., [40 41]). So far, significant gains in usability have been achieved, such as the recent addition of cognitively adequate landmarks to route planners $|16|$. Given this progress, the present paper argues that our focus can turn to other issues, such as the basic function of WAs. This is due to observations that WAs may have effectively become too usable, such that navigators are depending on them to the detriment of their geographic knowledge and orientation skills [33 42]. This paper therefore proposes a new direction for the development of WAs, where they be judged on the speed 
with which navigators become independent of them. Following a review of the relevant literature, this paper presents an implementation of our proposal and reports on the results from testing these ideas.

\section{Background}

This section reviews some of the reasons why WAs have achieved such popularity, some disadvantages of current WAs, and a discussion of some initial attempts to address these problems. Using this information, a proposal is made for a new functionality of WAs.

\subsection{The advantages of present wayfinding aids}

We discuss three reasons why WAs have become so popular: (i) users no longer need to acquire geographic knowledge for route planning; (ii) they do not need to rely on their own capacity to maintain geographic orientation while traveling; and for both of these reasons, (iii) cognitive resources are released that can be used on other tasks.

The first reason for the popularity of WAs is that users do not need to learn any geographic knowledge for route planning [11|. This advantage can be illustrated with the example of London taxi drivers, a group of navigators famous for their extensive geographic knowledge. On average, "All London" taxi license candidates require four years to learn the streets and landmarks within only a six mile radius of the center of London, as well as a general understanding of the entire city [54|. This training also involves learning 320 prescribed routes and their associated landmarks. Such an arduous process can be contrasted with using a wayfinding aid, which can be commanded to produce, within seconds, route directions, points of interest, and a map for almost any city.

The vast travel range of modern locomotion aids compounds the problem of poor geographic knowledge because people are more often traveling outside familiar environments |11|. For example, the percentage of Germans vacationing outside Europe rose by at least 29 per cent between 1964 and 1992 due to such factors as cheaper airline travel |37|. Rather than the effortful, time consuming process of learning new geographic knowledge at the destination, such tourists can now rely on WAs such as personal navigation devices or free online maps.

A second reason for the popularity of WAs applies specifically to personal navigation devices (PNDs). Such systems can provide continual, precise geographic orientation updates or, more specifically, indications of present location and heading. Since humans are mobile animals, the threat of geographic disorientation has been present throughout their existence. Therefore, similar to other such organisms, humans have developed strategies by which to remain geographically oriented using their sensory and cognitive systems [32]. One strategy that humans employ is piloting. This involves using landmarks to find one's current position in the environment or on a map, which is stored in a WA or in the navigator's memory. Additionally, navigators can use dead reckoning. This involves monitoring sensory flow (mostly from proprioception, vision, and audition) to update rotation and translation of the body relative to the starting point [17|. PNDs, however, can be more reliable than these strategies in providing geographic orientation $[3 \mid$.

The greater reliability of WAs for geographic orientation can be illustrated using another contrast with a group of famous navigators, the Hai||om of Namibia. Members of the

www.josis.org 
Hai||om were often employed by European colonizers for their excellent geographic orientation ability [58|. In a field study comparing their pointing precision with a PND, however, their average error was found to be $16.4^{\circ}$ [58|. Interestingly, the average error of males, $19.1^{\circ}$, was $8.5^{\circ}$ worse than that of females. Another contrast can be made using the Inuit of Canada, a group respected for their ability to maintain geographic orientation without wayfinding aids, even on long journeys across barren areas of the Arctic [3]. Like London taxi drivers and the Hai ||om, the skills of the Inuit take a number of years to develop, and they are fallible. Inuit hunters are particularly likely to become geographically disoriented during a fog $[3 \mid$. This disables visual means of orientation, potentially fatal given the harsh environment. As opposed to the Hai||om, who view PNDs as inefficient [58|, and London taxi drivers, who must perform without them [54], PNDs have become popular with the Inuit because they save time and reduce risk in fog $|3|$.

The third reason for the popularity of wayfinding aids follows from the above arguments about reliance upon them instead of spending many years collecting geographic knowledge and learning geographic orientation strategies. The need for environmental engagement reduces when using WAs [3|. Here, users can depend on the device and focus their limited visual processing resources [47| on other situational aspects. Hence, the cognitive resources usually captured by this engagement are released and can be devoted, for example, to increasing driver safety $[29 \mid$. Here, instead of constantly monitoring a map and searching for prominent environmental features, drivers can devote their attention to enhancing vehicle control, awareness of other road users, and traffic hazards $|29|$. With these significant reductions in required geographic knowledge, geographic orientation skills, and cognitive resources for wayfinding, it may therefore appear that the development of WAs has been a resounding success. A number of recent studies, however, challenge this claim.

\subsection{The disadvantages of present wayfinding aids}

As predicted [9] early in the development of personal navigation devices (PNDs), poorer development of geographic knowledge has been found in those using PNDs compared to other types of support, such as paper maps [3.7.25.30 39.59|. Additionally, at least one study has found reduced development of geographic orientation skills in PND users [3]. One of the earliest studies showing a decrease in development of geographic knowledge used a virtual environment and mimicked turn by turn instructions of a typical PND [7]. It revealed that those who had prepared for travel along the route by studying a map, taking notes, and using the map whilst moving, were able to produce maps that were more representative of the task area than PND users.

Whilst these findings of poorer geographic knowledge and orientation skills have led to concerns about device dependency, one investigation of PND use amongst novice Spanish taxi drivers found that most became essentially independent of the devices after a period of learning [19]. Note, however, that these findings may not apply to the general population since navigation is a constant activity of taxi drivers. Also, there is the possibility that independence may occur more slowly than for traditional wayfinding assistance, such as paper maps, due to automation of the wayfinding process in PNDs.

This automation of wayfinding and orientation by personal navigation devices causes dependency because users reduce their engagement with the environment |3.30|. More specifically, this disengagement reduces the depth of cognitive processing required to com- 
plete wayfinding tasks $[6]$. With shallower processing of the environment, there are fewer opportunities to develop good geographic knowledge and geographic orientation skills via such processing. For example, a user that is engaged with the environment through which they are navigating will have more chances to memorize nearby landmarks and practice the skill of constantly updating their alignment with global references such as cardinal directions.

The notion of poor environmental engagement during PND use is supported by the results of the study considered previously [7]. Here, PND users included fewer landmarks in their hand drawn maps of the area, "remembered fewer scenes [and] were less accurate in their ordering of images seen along routes" [7. p. 414]. This greater dependency is a paradoxical disadvantage because it will cause difficulty in the event of device malfunction [3.39| and is troubling from the perspective of user empowerment [24|. Given this problem of reliance on WAs, they could be improved by adding a training function. A successful implementation of this may reverse the present trend, instead offering navigators a chance to increase their independence through using such a device.

\subsection{Prompting environmental engagement}

Further development of present WAs could address the disadvantages identified above by prompting the user to engage more with their surroundings. We will discuss two ways of prompting. First, we will argue that adding landmarks to automatically generated route directions may appear to produce such engagement, although there are some limitations caused by the conciseness of this information. The second way is to turn the attention of the user back into the environment by omitting constant orientation updates.

\subsubsection{Reducing dependency by adding landmarks}

Wayfinding aids now provide route directions that include landmarks at decision points [16|. This type of improvement has been recommended throughout almost the past two decades [2.6.31] as a way to reduce the system-user gap [40]. This gap is created by information systems (in this case WAs) representing and processing information in a different way to that of psychological systems, such as found in humans [35|. When these two systems are set to work on the same problem, modifications are required to either, or both, in order to bridge this gap and enable them to work together. The turn-by-turn descriptions of typical WAs provide a good example of the system-user gap. These lack references to landmarks which humans, who often use the piloting strategy to maintain geographic orientation, generally require for successful navigation [31|. Consequently, landmarks are always mentioned when humans give verbal directions to others [14 15.31| and human navigation performance suffers when route directions are only based on street names and distances $\mid 52$ 53|.

Given that landmarks are particularly noticeable features of an area, one may consider that their inclusion in route directions would increase environmental engagement and thereby spatial knowledge. Discussion of this possibility elsewhere [6|, however, highlights the issue that landmarks are presently seen as merely a way to increase ease of use for WAs (see, e.g., [41|), not geographic knowledge for the area. Despite this, it is also reasonable to propose that a set of directions with integrated landmarks, drawing a user's attention to them at each turn, would at least produce more complete geographic knowledge than a

www.josis.org 
set only based around street names and distances. This approach of seeking a compromise may be the most effective path to take in retaining the cognitive resource gains of WA use whilst avoiding the lack of geographic knowledge and orientation skill development that is occurring with current devices. Training, however, is a more direct approach to increasing the acquirement of geographic knowledge.

\subsubsection{Active training}

At least one direct attempt has been made at developing a personal navigation device that produces relatively higher geographic knowledge in users [39|. The premise of the study was that because PNDs currently provide constant position updates, users disengage from the geographic orientation process and wait for this information to be delivered to them. This was described as putting users "out of the control loop" |39. p. 240], although the concept is explained more clearly elsewhere [42]. Here, the distinction between adaptive and adaptable systems is highlighted.

Since present PNDs provide constant orientation updates, they are a good example of an adaptive system, which essentially adapts itself to the changing needs of the user. The proposal of [39] was instead one of an adaptable system, where users adapt it to their needs by, in this case, requesting orientation updates as needed. Although requiring more work by users, it has been found that an adaptable system produces greater learning of geographic knowledge than an adaptive system [42|.

The adaptable system of [39] had two elements. In addition to position updates only being provided to navigators on request, position quizzes also occurred on a random basis throughout the task. This was done in order to prompt engagement with the environment, by which navigators could attempt to produce the position updates themselves. Compared to those given automatic position updates (adaptive system), those who were more environmentally engaged (via the adaptable system) displayed better performance when subsequently navigating through the same environment unaided [39|. These participants also produced better estimations of the relative directions to various targets in static scenes from the environment to which they had just been exposed, an effect observed elsewhere [42].

An important limitation of [39], however, is that the cognitive capture of the modifications to increase environmental engagement was not quantified. This could have been measured by adding a hazard avoidance component to the navigation task. The utility of the modification proposed in [39| would also be easier to judge by contrasting its level of cognitive capture with that of traditional wayfinding using paper maps and the like. Nevertheless, the study shows that a PND can be modified to produce the benefits of greater environmental engagement, whilst still providing precise position updates if needed. In this way, it appears that the independence of navigators can be promoted and [42] confirms this since it is based on user testing of currently available PNDs.

\subsection{The dual strategy for geographic orientation}

The studies reviewed above, concerning ways to train PND users to improve their geographic orientation skills, have focused on the piloting strategy for geographic orientation. This is because of the emphasis on adding landmarks to wayfinding aids such as online maps [45] and the location updating emphasis of [39| and [42]. As mentioned previously, however, humans can also deploy a dead reckoning strategy, which is based on updating 
one's heading relative to a known point (such as the origin of travel), or a globally available reference such as a cardinal direction. Furthermore, it is a common finding of studies investigating sex differences in spatial performance that those who put both of these strategies to use make fewer errors and reach destinations faster [10,43,44]. Whilst these studies have revealed that males favor the dual strategy more than females, the following descriptions of this section of the literature omit the emphasis on sex differences. This is because the purpose of the present study was to apply these findings in the pursuit of an improved wayfinding aid. The abilities of direction pointing and sense of direction, covered in studies discussed next, have both been described as underpinning the ability of dead reckoning.

\subsubsection{Pointing precision studies}

Measurements of pointing precision often favor those that typically have a dual strategy for geographic orientation. These studies measure an individual's precision in pointing to a presently out of sight, but previously visited point [23|. Despite the field study of Hai||Om participants mentioned above, it is generally found that males, or those who tend to use both landmarks and dead reckoning for spatial orientation, are more precise in pointing tasks [12]. The review of [12| also observed that it was only the easier pointing tasks that produced no performance difference between those normally relying on piloting and those with a more versatile approach. An example of an easy task is when testing occurred in a campus setting with which participants were already familiar. Here it was hypothesized that task difficulty could be expressed as the level of visuo-spatial working memory (VSWM) load, since the capacity of this type of memory is greater in males, or those usually found to rely both on dead reckoning and piloting [12|. This hypothesis prompted the use of multiple levels of difficulty in the present study.

\subsubsection{Sense of direction studies}

Pointing precision has also been described as an expression of a higher-order ability, an individual's sense of direction (SOD) [5,23,48]. At least one self-report study of SOD reflects the above difference in pointing precision, where those usually found to have a dual strategy for orientation delivered higher ratings of their SOD than landmark strategy users [13]. Another study, however, found no such difference in self-report SOD [36|. In considering the definition of SOD, its importance for versatility in maintaining geographic orientation appears to be clear.

A sense of direction is defined as "knowledge of the body's facing direction relative to a stable spatial framework anchored to the environment (i.e., its allocentric heading)" [50 p. 516]. An example of such a framework is the set of cardinal directions [22|. The direction that the body is going in, or heading, can be determined by sensing the angle between forward motion and a constant direction within one's surroundings, such as north [49]. Thus, an individual's sense of direction can be measured by the degree of precision in revising, or updating, the individual's heading in response to changes in the direction that they face [50|. In this way it appears to contribute to the process of dead reckoning. Given that the route directions produced by those that are usually found to deploy a dual strategy for geographic orientation contain more frequent references to cardinal directions, whilst simultaneously mentioning fewer landmarks $[8.15 .27 .55 \mid$, the cause may be their having a greater sense of direction. Using the Santa Barbara sense of direction scale (SBSOD), such an advantage of males was also expected to be found in this investigation.

www.josis.org 


\subsection{The present study}

The above findings of poorer orientation versatility in some navigators suggest that wayfinding aids, and personal navigation devices in particular, could be designed as training tools to produce more flexibility for geographic orientation in such individuals. Given that some people appear not to be as precise as others in providing heading updates for themselves, PNDs could be modified to provide these updates. Such an implementation would be an example of an adaptive approach, where an adaptable version would only supply updates on demand. The present study tested the former approach in order to establish whether simply providing participants with reliable heading information produced evidence of a dual strategy in those usually relying only on landmarks. If such an effect was observed, this would support the development of an adaptable system that might train navigators to be self-reliant in heading updating and thereby benefit from the versatility of a dual strategy for geographic orientation.

To the authors' knowledge, no prior investigators have attempted to measure the effect of providing navigators with precise, constant heading updates in the form of cardinal directions via PND-type interface. The idea itself, however, is not new. A number of studies [20-22| have identified this as a much needed addition to navigation displays in order to assist in the difficult task of making cardinal direction judgments when the map is misaligned with a forward facing view. These authors do not, however, appear to have tested the outcome of their suggestion. Additionally, the provision of automatic cardinal direction updates by a PND has been referred to as easy to accomplish $[41 \mid$ and at least two popular PNDs provide them in an adaptive format $[4.34 \mid$.

\section{Method}

This was a 2 (participant sex: male, female) $\times 3$ (type of spatial reference information: heading updates, landmarks, control) between participants experimental design. Participants were randomly allocated to one of the three spatial reference conditions. The latter factor could have been within subjects, however, this was avoided due to the problem of having to produce three different route stimuli (see Section 3.1) of comparable complexity. The dependent variable was the degree of correct recall for the route in the video. All participants took the Santa Barbara sense of direction (SBSOD) scale after the map judgment task, so as to avoid any influence on performance arising from exposure to the scale.

\subsection{Route video}

All participants viewed the same silent 3:03 minute video, although different textual information appeared on screen in the landmarks and heading updates conditions. The video involved a car being driven at an average of $20 \mathrm{~km} / \mathrm{h}$ through $979 \mathrm{~m}$ of suburban streets in Melbourne, Australia. The route of the video is presented in Figure 1. The videos can be accessed at $|57|$.

The landmarks video contained a text highlight displayed for five seconds at each turn and at the destination, comprising the name of a landmark at that location and an arrow pointing to it. According to the classification of $[41 \mid$, all except two were visual landmarks (various shop fronts and houses), whilst the fourth (T-junction) and sixth (roundabout) were structural landmarks. The heading updates video used text in the same fashion although 


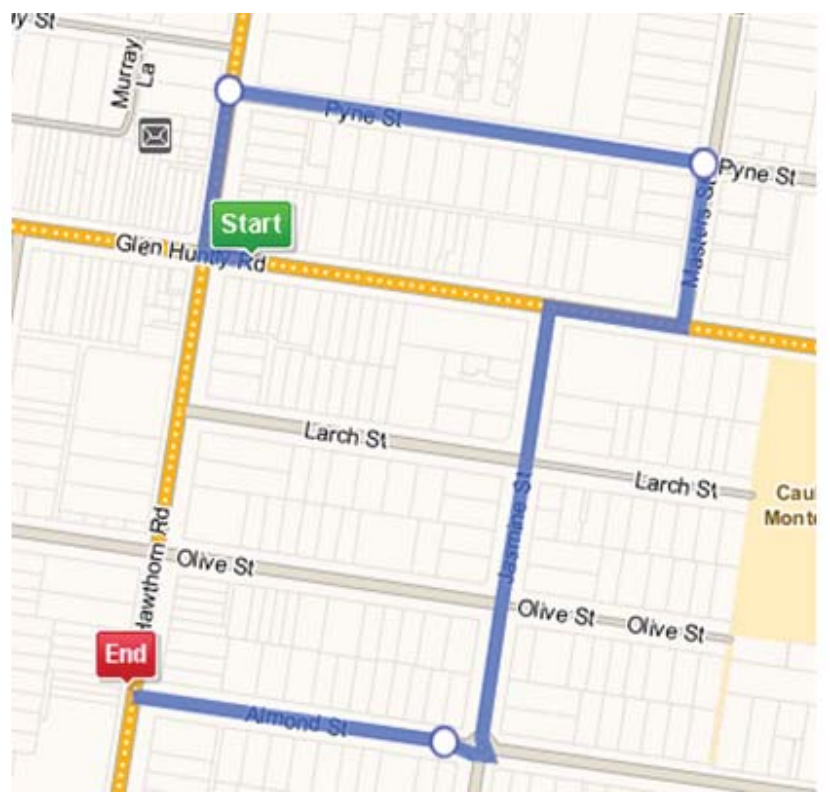

Figure 1: Route of the video [46|.

here the relevant cardinal direction was shown as soon as each straight section came into view. The control video did not contain any superimposed information.

\subsection{Route maps}

The main outcome measure of the study was participants' judgments of six route structure maps (examples in Figure 2 full set in Appendix 1 of [56|). Of the six maps, one was the correct map whilst the others contained errors. Following Hegarty et al. [23 experiment $5]$, the error types were the number of route segments (topological errors), direction of turns (relative direction errors), and length of route segments (metric errors). These three error types were listed under each map in the questionnaire, with participants indicating that the map was correct by leaving all check boxes next to the items empty, or checking appropriate boxes for incorrect maps (questionnaire available in Appendix 1 of [56| and as a supplementary file for this article, see doi:10.5311/JOSIS.2011.3.43). Three of the maps contained one error each and two of them contained two errors each.

As shown in Figure 2, the heading updates maps contained a compass rose and were oriented toward north, whereas the maps of the other conditions were oriented such that the first segment was directed upward on screen. The heading updates maps were configured in this way so that they would correspond with the compass directions given in the video. It was reasoned that the disadvantage of having the first segment heading in a counterintuitive leftward direction would be balanced by the participant's recall of the appropriate cardinal direction of that segment.

Producing maps with these specific types of errors enabled testing of participants' knowledge of the route at multiple levels of difficulty. Thus we could test the hypothe- 

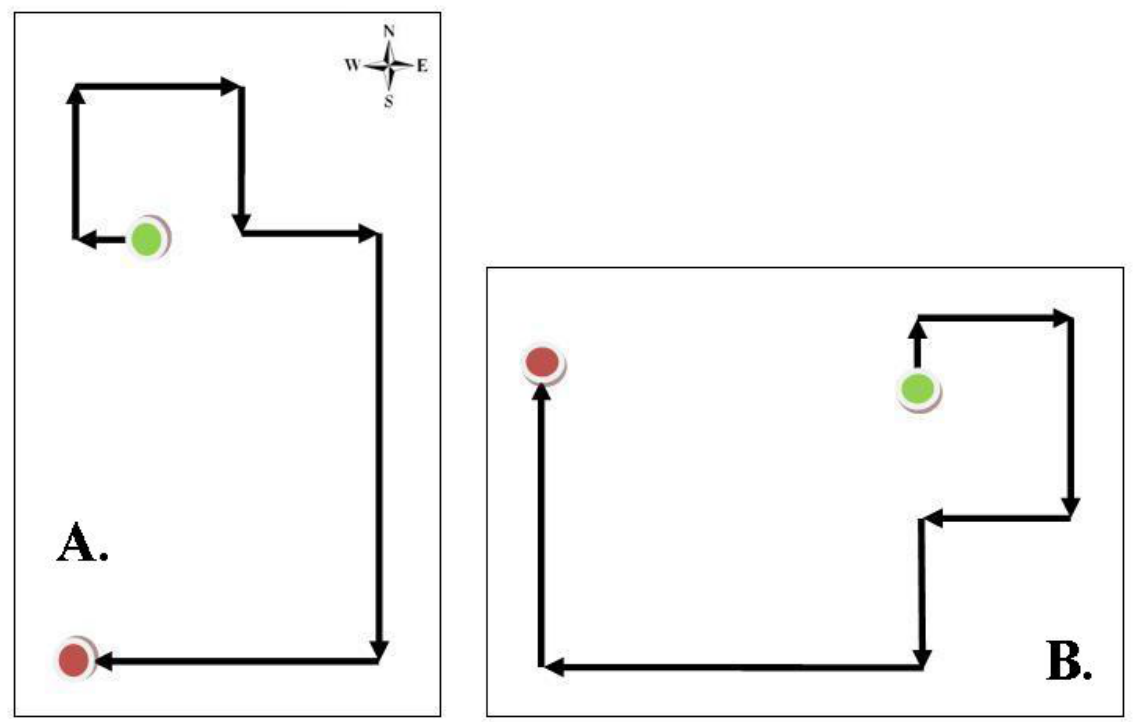

Figure 2: Example of a route structure map with relative direction error (the fourth turn should have gone to the right). A. was used in heading updates condition, B. was used in landmarks and control conditions.

sis that a sex difference in spatial performance for environmental scale tasks only emerges at higher difficulty levels |12|. Here, the material enabled the development of three difficulty levels - the first of which was judging whether or not each map was correct. The next level of difficulty was judging what specific type of error was displayed in the maps with a single error, whilst the most difficult was correctly diagnosing the maps with two errors.

\subsection{Manipulation check}

As a manipulation check, participants' knowledge of the sequence of landmarks or heading updates was also tested. For the former, they were to match a list of 11 landmarks (containing four that were not in the video) with a list of seven turn numbers. Similarly, those in the heading updates condition matched a list of eight cardinal directions (also containing four that were not seen in the video) with a list of seven straight segment numbers.

\subsection{Sense of direction test}

The Santa Barbara sense of direction (SBSOD) was used to check participants' sense of direction because the tasks of the present study may have involved this ability. This selfreport measure contains 15 items with an internal reliability of $0.88|23|$. Each is a Likerttype scale with anchors " $1=$ strongly agree" and " 7 = strongly disagree" (see [23| for the list of items). 


\subsection{Procedure}

The procedure was conducted online (see |57|). This was done in order to collect a large sample, given that many spatial cognition studies have poor statistical power due to small sample size (e.g., [7 26, 52,53|). A discussion of the limitations of an online procedure is found in Section 5.4 After a brief practice trial to familiarize participants with the process of judging a set of maps based on a separate route video, each participant watched the experiment video of their condition. This was followed by a questionnaire containing the route maps, the appropriate manipulation check, and then the SBSOD. Participants were only asked to report their sex at the end of the study, since simply drawing participants' attention to their sex has been found to alter spatial performance [28,38|.

\section{Results}

There were 124 participants in the study, although six were excluded as they did not finish their questionnaires. Only 113 participants chose to indicate their age, and the range was 18 to 90 years with a median of 21 (st. dev. $=10.27$ ). The age distribution was strongly positively skewed, with $80 \%$ of participants aged less than 30 years. Three extreme outliers (aged 56 and above) were excluded from the results. This was due to a negative relationship between age and both piloting and dead reckoning performance [1|. Note, however, that their exclusion did not significantly alter the results. As shown in Table 1 participants were relatively evenly distributed across conditions and sexes.

\begin{tabular}{l|rrr|r} 
& Control & Landmarks & Heading updates & Total \\
\hline Male & 17 & 15 & 19 & 51 \\
Female & 22 & 24 & 18 & 64 \\
Total & 39 & 39 & 37 & 115
\end{tabular}

Table 1: Participants by sex and condition

\subsection{Manipulation checks}

Manipulations were checked through calculating the Levenshtein edit distance between the sequence of spatial references supplied by participants and that of the video. This involved counting the number of substitutions, insertions, or deletions from a reference sequence [18|, such that a lower score meant the supplied sequence was closer to the reference. This method of checking the manipulation reflected the use of a sequence of spatial references for wayfinding. Since the heading updates condition contained one cardinal direction for each of the seven straight route segments, here the reference string was composed of seven characters identifying the relevant directions. Eight participants were excluded from the heading updates manipulation check because they stated that they did not see any headings indicated in the video. An independent samples $t$-test revealed that males scored slightly better $($ mean $=0.60$, st. dev. $=1.60)$ than females $($ mean $=1.14$, st. dev. $=$ $1.70)$, although this difference was not significant $(t(27)=-0.87, p=0.38)$. The effect size (mean difference $=-0.54,95 \%$ CI: -1.80 to 0.71$)$ was small $\left(\eta^{2}=0.02\right)$. Note that both edit distances were minimal, hence this manipulation was successful.

www.josis.org 
A comparison of landmark sequence knowledge could be made between conditions since they were merely highlighted in the landmarks condition, but still visible in the videos of the other conditions. Since there were four false landmarks in addition to the seven occurring at turns and the end of the route, the reference string contained 11 characters with zeros representing landmarks specified by participants as being fake. The characters were arranged by the order in which landmarks were listed in the questionnaire. A number of participants in each condition stated that they did not notice landmarks in the videos, such that 10 were excluded from the control condition, 3 from the landmarks condition, and 12 from the headings condition.

A 2 (participant sex: male, female) $\times 3$ (type of spatial reference information: control, landmarks, heading updates) analysis of variance (ANOVA) was conducted with landmark sequence Levenshtein edit distance as the dependent variable. This revealed a significant main effect of condition $(F(2,78)=22.04, p<0.001)$, the effect size being large (calculated using eta squared), 0.35 . Tukey's HSD post-hoc comparisons revealed that edit distances in the landmark condition (mean $=3.53$, st. dev. $=2.23$ ) were significantly lower, or better, than both the control $($ mean $=5.86$, st. dev. $=1.64)$ and the heading update conditions (mean $=7.16$, st. dev. $=1.98)$. There was no significant difference in edit distances between the control and heading update conditions. Again, no sex difference was found $(F(1,78)=$ $1.04, p=0.31, \eta^{2}=0.01$ ), with males (mean $=5.58$, st. dev. $=2.60$ ) scoring slightly worse than females $($ mean $=4.88$, st. dev. $=2.41)$. Overall, the manipulation was successful as the Levenshtein edit distance in the landmark condition was significantly smaller than the other conditions.

\subsection{Map judgment tasks}

The results for the map judgment tasks can be summarized in order of increasing difficulty of task.

\subsubsection{Low difficulty: Route matching task}

A 2 (participant sex: male, female) $\times 3$ (type of spatial reference information: heading updates, landmarks, control) ANOVA was performed with number of correct route match judgments as the dependent variable. This measured participants' ability to detect simply whether or not the map matched the route, without having to specify what was wrong with incorrect maps. A perfect score on this measure would have been to check any error check box for all of the maps apart from leaving them empty for the correct map. No significant main effects of condition $\left(F(2,109)=0.09, p=0.92, \eta^{2}<0.01\right)$ or $\operatorname{sex}(F(1,109)=0.02$, $\left.p=0.90, \eta^{2}<0.001\right)$ were found, and the interaction between sex and condition was likewise non-significant $\left(F(2,109)=0.03, p=0.97, \eta^{2}<0.001\right)$. The mean number of correct judgments for all participants was 5.38 (st. dev. $=1.06)$ out of six.

\subsubsection{High difficulty: Specifying the fault within each map}

Participants' ability to specify the precise fault in each route map, a more difficult task than the above, was also investigated. A second $2 \times 3$ ANOVA using the same factors revealed no effect of condition $\left(F(2,109)=0.18, p=0.84, \eta^{2}<0.01\right)$ or $\operatorname{sex}(F(1,109)=$ $\left.0.004, p=0.95, \eta^{2}<0.001\right)$. There was also no significant interaction between male and 
female performance $\left(F(2,109)=0.08, p=0.93, \eta^{2}<0.01\right)$. The mean number of correct judgments for all participants was 2.49 (st. dev. $=1.39$ ) out of five, the correct map being excluded from this analysis.

\subsubsection{Single fault and double fault maps}

A further level of difficulty was available in the two maps containing two faults. Performance on these was compared with the three single fault maps. A one-way between groups multivariate analysis of variance (MANOVA) was used to investigate sex differences in responses to the single and double fault maps. This revealed a non-significant difference between males and females, $F(1,112)=0.02, p=0.98$, Wilk's $\lambda=1.00, \eta^{2}<0.001$. The mean number of correct judgments for all participants regarding single error maps was 1.98 (st. dev. $=1.07$ ) out of three, whilst for double error maps it was $0.50($ st. dev. $=0.64$ ) out of two.

\subsection{Use of spatial reference information}

Given that performance on the map judgment tasks did not appear to differ by spatial reference condition, participants' reported use of such information was examined. Here it was found that $86 \%$ of participants reported using heading updates information in the map judgment task. Of those who did use heading updates, there was a non-significant difference in the rate of usage between males (93\%) and females (79\%), $p=0.27$, one tailed Fisher's exact test. Usage of cardinal direction information could not be compared across conditions as it was only given to those in the heading updates condition.

It was possible to compare the use of landmarks when judging the maps across conditions since landmarks were merely highlighted in the landmarks video. A fault of the questionnaire, however, meant that usage of landmark information was not collected for the heading updates condition. It was found that $34 \%$ of participants reported using landmarks information. Of those that did use landmarks, a chi-square test for independence (with Yates' continuity correction) revealed a non-significant difference between those in the control $(31 \%)$ and those in the landmarks $(36 \%)$ conditions, $\chi^{2}(1,65)=0.03, p=0.87$, $\phi=-0.05$. Likewise, a chi-square test for independence (with Yates' continuity correction) revealed that, for those using landmarks, there was a non-significant difference in the rate of usage between males $(42 \%)$ and females $(30 \%), \chi^{2}(1,65)=0.56, p=0.46, \phi=0.13$.

The above results show that the rate of using landmarks was not high in this study. This goes against findings in other studies which show a high preference for, and benefit of, landmarks in navigation tasks. The relatively higher use of cardinal directions, compared to landmarks, is considered further in Section 5.

\subsection{Sense of direction}

In a one-way ANOVA with Santa Barbara sense of direction (SBSOD) scale scores as the dependent variable and sex as the factor, a significant difference between males (mean $=$ 4.85 , st. dev. $=0.88)$ and females $($ mean $=4.21$, st. dev. $=1.13)$ was found $(F(1,113)=11.04$, $\left.p<0.01, \eta^{2}=0.10\right)$. The practical significance of this difference was low, however, since the average male SBSOD score was only $9 \%$ higher than that of females.

www.josis.org 
The Spearman coefficient was used for the route matching task. This revealed a moderate, positive, non-significant correlation ( $\rho=0.14, n=115, p=0.13)$ between sense of direction as measured by the SBSOD and number of correct judgments on the route matching task. A Pearson product-moment correlation coefficient was calculated for the map fault task. This revealed a small, positive, non-significant correlation between SBSOD and number of correct fault identifications for all maps, $\rho=0.11, n=115, p=0.23$.

\section{Discussion}

The aim of the present study was to determine whether greater navigation performance would occur in participants that were given the spatial reference information which is used in the two wayfinding strategies of piloting and dead reckoning. A further aim was to determine whether those that usually rely on a landmark strategy for geographic orientation would respond more significantly to the provision of heading updates than those with a dual strategy. This is because prior research appears to show that some navigators' ability to provide heading updates for themselves is not as precise as others, reducing their versatility in gaining geographic orientation. This was done in order to investigate a possible avenue for the improvement of wayfinding aids (WAs) such as personal navigation devices (PNDs).

The hypothesized benefit of providing either landmark or heading updates over the control group was not supported. Likewise, the hypothesized greater response of females (those who more often appear to use a landmark strategy) over males (more frequently found to use a dual strategy of landmarks and dead reckoning) to the provision of heading updates was not supported. Additionally, the hypothesis of greater task difficulty leading to a greater sex difference favoring males was not supported. In contrast, the hypothesized greater sense of direction (SOD) of males over females was supported. Despite this outcome, the hypothesized expression of this better SOD in a higher number of correct map judgments amongst males was not supported.

\subsection{Manipulation checks}

The first means of explaining this lack of effect of the spatial reference conditions over control is to appeal to a manipulation failure. As shown in the results, however, participants displayed good recall for the relevant sequence of references in both the heading updates and landmarks conditions.

Since there was no difference in performance between levels of task difficulty, one may also consider a manipulation failure for this factor. The least difficult type of judgment was to determine whether or not each map correctly represented the route, without having to specify the reason. The argument that this was easier is supported by the results, where $90 \%$ of participants' judgments were correct and no sex difference was found. The next level of difficulty was to determine the specific fault of each map. That this was more difficult is also supported by the results, where only $50 \%$ of participants' judgments were correct. Interestingly, however, no difference in performance was found between those typically using a dual strategy for orientation as opposed to a landmark strategy.

The task of determining the specific error in the map yielded a further level of difficulty. Two of the six maps contained two errors, whilst three contained only one (the correct 
map, containing no errors, was excluded from the analysis). Whilst the results indicated that judging the double error maps was more difficult (25\% correct) than the single error maps (66\% correct), again no performance difference emerged in the hardest version of the task. Assuming that these levels of difficulty corresponded with increasing load on visuospatial working memory, the hypothesis [12| that a male advantage in large scale spatial orientation emerges only for difficult tasks is therefore not supported by this set of results.

Another attempted manipulation was participants' level of spatial orientation. Since the procedure was thought to activate strategies of piloting and dead reckoning so that participants might maintain orientation, the task needed to present a disorientation threat. It appears, however, that it possibly did not, from two perspectives. The first manner in which the present procedure may not have threatened participants with disorientation is that they watched someone else deploying geographic orientation strategies whilst navigating along an urban route. Participants, themselves, may not have been required to deploy such strategies as they were instead watching the product of the strategies in the shape of the route taken by the navigator during the video. Participants could have attended to references such as landmarks, but this would not have been to avoid becoming lost and failing to reach a destination. They might have been primed for this more passive approach from the practice task, the theme of which was matching the three dimensional shape of the video route with the two dimensional shape in the maps. Incidental email feedback from one participant attests to this:

"I started 'drawing' a visual map in my head that looks the [sic] like the maps shown in training because I knew that's what I had to do."

The outcome measure of judging the maps may also have lacked the possibility of participants becoming disoriented. It is possible that this map judgment task was instead simply a shape judgment task. These may not have been maps, but more pictures of directed lines of a particular shape, an idea supported by the definition of a map. Maps present "spatial information as an allocentric representation of the environment" [11 p. 217]. Whilst the line pictures could have performed this function and could be conceived of as having had an allocentric perspective, they were highly abstract and there were no surrounding streets or choice points at intersections from which participants might pick out a route. Hence, in both the video task and the "map" (read "shape") judgment task, the need for geographic orientation skills may have been absent. In this way there was possibly no need to deploy the geographic orientation strategies to which the spatial references of the landmarks and heading updates conditions pertained.

\subsection{Use of spatial references}

Participants' rate of using the spatial references provided mixed support for the charge that the procedure failed to threaten participants with disorientation. Most (86\%) of those in the heading updates condition stated that they used such references in the "map" judgment task. In contrast, 20\% (landmarks condition) and 14\% (control condition) stated that they used landmarks in judging the "maps." In terms of landmarks, it appears that the widespread support in the literature for the idea that they are vital for wayfinding is not reflected in the present study, since the majority of participants stated that they did not use them. This is more comprehensible when considering it as a shape recognition task instead 
of a wayfinding task. If a new version of the task were devised which incorporated a disorientation threat where participants must work to ensure they reach a particular destination, perhaps this landmark usage rate would increase.

In opposition to the rate of landmark usage, the majority of those receiving heading updates stated that they used them. As noted above in Section 3. this may have been due to the confounding effect of the misaligned maps in the heading updates condition, where the first segment went to the left rather than a more intuitive upward direction. Perhaps the high usage of the heading updates spatial reference type reflects participants' use of the cardinal directions to align the map, not to recall the shape of the route. Alternatively, since the sequence of cardinal directions can be used to trace a line shape, perhaps the higher usage of this information reflects its being a shape recall task. This possibility, however, was not expressed in terms of higher map judgment performance in the heading updates group.

\subsection{Sense of direction}

One may also account for the lack of influence of sense of direction (SOD) on "map" judgment by appealing to the low disorientation threat of the procedure. Recall that the definition of SOD is to estimate the direction of one's heading relative to a stable spatial framework such as the cardinal directions. Perhaps participants did not require such a facility on this task as there was no need to sense their direction in this way. More succinctly, SOD may be of no use in abstracting a two dimensional line shape from motion through a three dimensional scene. Here, rather than sensing direction, participants may have been comparing or updating their direction continuously with reference to a past direction that they had taken, rather than a constant direction in the environment, in order to understand the shape of the route.

A similar explanation can be furnished for the absence of any influence of the levels of task difficulty on sex differences. The hypothesis of [12| regarding this advises that the task must be about spatial orientation in large scale space. Despite the urban streetscape content of the video, this experiment does not appear to have contained such a task. Hence it may not have produced a visuo-spatial working memory load comparable to difficult environmental scale spatial orientation tasks of previous studies.

\subsection{Limitations of the online procedure}

Whilst conducting this experiment online is likely to have produced the large sample, this approach could have led to poor standardization of the procedure. For example, participants may have ignored the multiple messages throughout the trial to participate without assistance from others. Extraneous variables such as auditory distractions may also have been introduced through participants taking part in multiple, uncontrolled settings. Technical limitations of running trials online also reduced the scope of the study. For example, participants' drawings of the route, an approach used elsewhere [23|, may have produced a richer measure of recall. Last, the sedentary nature of the procedure may have contributed to the lack of deployment of geographic orientation strategies. Here, the present procedure contrasts strongly with [51|, for example, where participants walked through woods and had to find their way back to the origin. That the results of this experiment did not 
match those of others conducted in a more realistic setting perhaps attests to the ecological validity of such prior studies.

\section{Conclusion}

Current personal navigation devices (PNDs) do not appear to foster the development of better geographic knowledge and orientation skills amongst users. The study addressed this by extending prior work on an adaptive mode of providing orientation updates to such users. A review of the human wayfinding literature revealed a positive association between navigation performance and the more versatile approach of using both landmarks and dead reckoning to maintain orientation. This prompted the suggestion that PNDs support users' sense of direction via the provision of heading updates, in addition to the location updates already proposed elsewhere. An experiment testing the utility of heading updates did not appear to provide a clear assessment, possibly because the procedure did not threaten participants with geographic disorientation. Adequate testing of this promising new direction for the development of PNDs may require trials that involve locomotion in a real or virtual environment.

Due to participants not appearing to deploy geographic orientation strategies, this study cannot be used to answer the question of whether or not the provision of adaptive heading updates will induce a dual orientation strategy. The study does, however, provide a basis for future work where the route video may be supplanted by a real world or virtual environment task requiring participants to find their way to a destination. This may produce a need for participants to use such strategies since performance on the task would require the avoidance of getting lost, rather than simply recalling the shape of the route. The study is therefore important for the indication that a navigation task must involve some level of disorientation threat. This is useful information for those wishing to target geographic orientation abilities in future studies.

\section{Acknowledgments}

The authors express their gratitude to the three anonymous reviewers for their assistance in improving the paper.

\section{References}

[1] Allen, G., Kirasic, K., Rashotte, M., And Haun, D. Aging and path integration skill: Kinesthetic and vestibular contributions to wayfinding. Attention, Perception, $\mathcal{E}$ Psychophysics 66 (2004), 170-179. doi:10.3758/bf03194870.

[2] Alm, H. Human factors considerations in vehicle navigation aids. Belhaven Press, London, UK, 1993.

[3] ApOrTA, C., AND Higgs, E. Satellite culture: Global positioning systems, Inuit wayfinding, and the need for a new account of technology. Current Anthropology 46 (2005), 729-753. doi:10.1086/432651

www.josis.org 
[4] APPLE INC. iPhone: Maps + compass. http://www.apple.com/iphone/features/ maps-compass.html, 2010. Retrieved 30 November, 2010.

[5] BELL, S., AND SAUCIER, D. Relationship among environmental pointing accuracy, mental rotation, sex, and hormones. Environment and Behavior 36 (2004), 251-265. doi: $10.1177 / 0013916503251470$

[6] Burnett, G. "Turn right at the traffic lights": The requirement for landmarks in vehicle navigation systems. The Journal of Navigation 53 (2000), 499-510. doi:10.1017/S0373463300001028

[7] Burnett, G., And LeE, K. The effect of vehicle navigation systems on the formation of cognitive maps. In Traffic and Transportation Psychology: Theory and Application, G. Underwood, Ed. Elsevier, Amsterdam, 2004, pp. 407-418.

[8] Charleston, S. The international wayfinding strategy scale: Evidence for crosscultural use with a sample from the UK. Perceptual and Motor Skills 106 (2008), 881-882. doi:10.2466/pms.106.3.881-882

[9] Chen, J., And StAnney, K. A theoretical model of wayfinding in virtual environments: Proposed strategies for navigational aiding. Presence: Teleoperators $\mathcal{E}$ Virtual Environments 8 (1999), 671-685. doi:10.1162/105474699566558

[10] Choi, J., McKillop, E., WARD, M., AND L'Hirondelle, N. Sex-specific relationships between route-learning strategies and abilities in a large-scale environment. Environment and Behavior 38 (2006), 791-801. doi:10.1177 / 0013916506287004

[11] ColucCia, E. Learning from maps: The role of visuo-spatial working memory. Applied Cognitive Psychology 22 (2008), 217-233. doi:10.1002/ acp.1357.

[12] Coluccia, E., AND Louse, G. Gender differences in spatial orientation: A review. Journal of Environmental Psychology 24 (2004), 329-340. doi:10.1016/j.jenvp.2004.08.006

[13] Cornell, E., Sorenson, A., AND Mio, T. Human sense of direction and wayfinding. Annals of the Association of American Geographers 93 (2003), 399-425. doi:10.1111/1467-8306.9302009.

[14] DANIEL, M.-P., AND DENIS, M. Spatial descriptions as navigational aids: A cognitive analysis of route directions. Kognitionswissenschaft 7 (1998), 45-52.

[15] DenIS, M. The description of routes: A cognitive approach to the production of spatial discourse. Cahiers de Psychologies Cognitive 16 (1997), 409-458.

[16] DUCKHAM, M., Winter, S., AND ROBINSON, M. Including landmarks in routing instructions. Journal of Location Based Services 4 (2010), 28-52. doi:10.1080/17489721003785602

[17] Fetsch, C., Deangelis, G., AND AngelaKi, D. Visual-vestibular cue integration for heading perception: Applications of optimal cue integration theory. European Journal of Neuroscience 31 (2010), 1721-1729. doi:10.1111/j.1460-9568.2010.07207.x

[18] GARCIA, E. Levenshtein edit distance. http://www.miislita.com/searchito/ levenshtein-edit-distance.html, 2006. Retrieved 14 May, 2010. 
[19] Girardin, F., AND Blat, J. The co-evolution of taxi drivers and their incar navigation systems. Pervasive and Mobile Computing 6 (2010), 424-434. doi:10.1016/j.pmcj.2010.03.002

[20] Gugerty, L., AND BROOKS, J. Seeing where you are heading: Integrating environmental and egocentric reference frames in cardinal direction judgments. Journal of Experimental Psychology: Applied 7 (2001), 251-266. doi:10.1037/1076-898X.7.3.251.

[21] GugERTY, L., AND BROOKS, J. Reference-frame misalignment and cardinal direction judgments: Group differences and strategies. Journal of Experimental Psychology / Applied 10 (2004), 75-88. doi:10.1037/1076-898X.10.2.75

[22] Gugerty, L., AND Rodes, W. A cognitive model of strategies for cardinal direction judgments. Spatial Cognition \& Computation 7 (2007), 179-212. doi:10.1080/13875860701418230

[23] Hegarty, M., Richardson, A., Montello, D., Lovelace, K., and Subbiah, I. Development of a self-report measure of environmental spatial ability. Intelligence 30 (2002), 425-447. doi:0.1016/S0160-2896(02)00116-2

[24] Hutchinson, A. Global impositioning systems: Is GPS technology actually harming our sense of direction? In The Walrus (2009). Retrieved 17 January, 2011, from http://www.walrusmagazine.com/articles/2009.11-health-globalimpositioning-systems/.

[25] Ishikawa, T., Fujiwara, H., ImAI, O., AND OKABE, A. Wayfinding with a GPSbased mobile navigation system: A comparison with maps and direct experience. Journal of Environmental Psychology 28 (2008), 74-82. doi:0.1016/j.jenvp.2007.09.002

[26] Jones, C., AND Healy, S. Differences in cue use and spatial memory in men and women. In Proceedings of the Royal Society B: Biological Sciences (2006), vol. 273, pp. 2241-2247. doi:10.1098/rspb.2006.3572

[27] Lawton, C. Gender differences in way-finding strategies: Relationship to spatial ability and spatial anxiety. Sex Roles 30 (1994), 765-779. doi:10.1007/BF01544230

[28] Lawton, C. Gender, spatial abilities, and wayfinding. In Handbook of Gender Research in Psychology, J. Chrisler and D. Mccreary, Eds. Springer, New York, 2010, pp. 317-341. doi:10.1007/978-1-4419-1465-1_16

[29] LEE, W.-C., AND CHENG, B.-W. Effects of using a portable navigation system and paper map in real driving. Accident Analysis \& Prevention 40 (2008), 303-308. doi:10.1016/j.aap.2007.06.010.

[30] Leshed, G., Velden, T., Rieger, O., Kot, B., And Sengers, P. In-car GPS navigation: Engagement with and disengagement from the environment. In Proceeding of the twenty-sixth annual SIGCHI conference on Human factors in computing systems (Florence, Italy, 2008), ACM. doi:10.1145/1357054.1357316

[31] Michon, P.-E., AND DENIS, M. When and why are visual landmarks used in giving directions? In Spatial Information Theory: Cognitive and Computational Foundations of Geographic Information Science, D. Montello, Ed., vol. 2205 of Lecture Notes in Computer Science. Springer, Berlin, 2001, pp. 292-305. doi:10.1007/3-540-45424-120 
[32] Montello, D. Navigation. In The Cambridge Handbook of Visuospatial Thinking, P. Shah and A. Miyake, Eds. Cambridge University Press, Cambridge, 2005, pp. 257-294.

[33] Montello, D. Cognitive research in GIScience: Recent achievements and future prospects. Geography Compass 3 (2009), 1824-1840. doi:10.1111/j.1749-8198.2009.00273.x

[34] NOKIA. Nokia 6210 Navigator, 2010. Retrieved 30 November, 2010, from http://www. nokia.com.au/find-products/all-phones/nokia-6210-navigator/specifications.

[35] Norman, D. Cognitive engineering. In User centred system design, D. Norman and S. Draper, Eds. Lawrence Erlbaum Associates, Hills Dale, NJ, 1986, pp. 31-61.

[36] Ohnishi, T., Matsuda, H., Hirakata, M., And Ugawa, Y. Navigation ability dependent neural activation in the human brain: An fMRI study. Neuroscience Research 55 (2006), 361-369. doi:10.1016/j.neures.2006.04.009

[37] Oppermann, M. Travel life cycle. Annals of Tourism Research 22 (1995), 535-552. doi:10.1016/0160-7383(95)00004-P

[38] ORTNER, T., AND SIEVERDING, M. Where are the gender differences? Male priming boosts spatial skills in women. Sex Roles 59 (2008), 274-281. doi:10.1007/ s11199-008-9448-9

[39] Parush, A., Ahuvia, S., AND EReV, I. Degradation in spatial knowledge acquisition when using automatic navigation systems. In Spatial Information Theory, S. Winter, M. Duckham, L. Kulik, and B. Kuipers, Eds. Springer, 2007, pp. 238-254.

[40] Raubal, M. Cognitive engineering for geographic information. Geography Compass 3 (2009), 1087-1104.

[41] Raubal, M., AND Winter, S. Enriching wayfinding instructions with local landmarks. In Geographic Information Science, M. Egenhofer and D. Mark, Eds., vol. 2478 of Lecture Notes in Computer Science. Springer, Berlin, 2002, pp. 243-259. doi:10.1007/3-540-45799-2_17.

[42] Richter, K.-F., Dara-Abrams, D., AND RAubal, M. Navigating and learning with location based services: A user-centric design. In Proc. 7 th International Symposium on LBS \& TeleCartography (2010), Springer, pp. 261-276.

[43] Sandstrom, N., Kaufman, J., And Huettel, S. Males and females use different distal cues in a virtual environment navigation task. Cognitive Brain Research 6 (1998), 351-360. doi:10.1016/S0926-6410(98)00002-0

[44] Saucier, D., Green, S., Leason, J., Macfadden, A., Bell, S., and Elias, L. Are sex differences in navigation caused by sexually dimorphic strategies or by differences in the ability to use the strategies? Behavioral Neuroscience 116 (2002), 403-410. doi:10.1037/0735-7044.116.3.403

[45] SENSIS. Landmarks in directions. http://www.whereis.com/help.htm?category=how, 2010. Retrieved 13 June 2011. 
[46] SENSIS. Whereis. http://www.whereis.com/, 2010. Retrieved 17 May 2010.

[47] SHAW, M., AND SHAW, P. Optimal allocation of cognitive resources to spatial locations. Journal of Experimental Psychology: Human Perception and Performance 3 (1977), 201-211. doi:10.1037/0096-1523.3.2.201

[48] SHOLL, M. The relation between sense of direction and mental geographic updating. Intelligence 12 (1988), 299-314. doi:10.1016/0160-2896(88)90028-1

[49] SHOlL, M. Human allocentric heading orientation and ability. Current Directions in Psychological Science 17 (2008), 275-280. doi:10.1111/j.1467-8721.2008.00589.x

[50] Sholl, M., Kenny, R., and Dellaporta, K. Allocentric-heading recall and its relation to self-reported sense-of-direction. Journal of Experimental Psychology: Learning, Memory \& Cognition 32 (2006), 516-533. doi:10.1037 / 0278-7393.32.3.516

[51] Silverman, I., Choi, J., Mackewn, A., Fisher, M., Moro, J., And OlshanSKY, E. Evolved mechanisms underlying wayfinding: Further studies on the huntergatherer theory of spatial sex differences. Evolution and Human Behavior 21 (2000), 201-213.

[52] TOM, A., AND DeNIS, M. Referring to landmark or street information in route directions: What difference does it make? In Spatial Information Theory, W. Kuhn, M. Worboys, and S. Timpf, Eds., vol. 2825 of Lecture Notes in Computer Science. Springer, Berlin, 2003, pp. 362-374. doi:10.1007/978-3-540-39923-0_24.

[53] TOM, A., AND Denis, M. Language and spatial cognition: Comparing the roles of landmarks and street names in route instructions. Applied Cognitive Psychology 18 (2004), 1213-1230. doi:10.1002/ acp.1045

[54] TRANSPORT FOR LONDON. Becoming a taxi driver. http://www.tfl.gov.uk/ businessandpartners/taxisandprivatehire/1406.aspx, 2010. Retrieved 21 May 2010.

[55] Ward, S., NewCombe, N., AND Overton, W. Turn left at the church, or three miles north: A study of direction giving and sex differences. Environment and Behavior 18 (1986), 192-213. doi:10.1177/0013916586182003

[56] WATERS, W. The relative benefit of reliable heading updates on urban wayfinding. $\mathrm{PhD}$ thesis, Thesis for Master of Applied Science (Geographic Information Systems), Department of Infrastructure Engineering, The University of Melbourne, 2010. http://repository.unimelb.edu.au/10187/8505.

[57] WATERS, W. Navigation experiment. http://www.boommap.com/start.html, Retrieved 2 January 2011.

[58] WIDLOK, T. Orientation in the wild: The shared cognition of the Hai ||Om bushpeople. Journal of the Royal Anthropological Institute 3 (1997), 317-332.

[59] Young, M., Stanton, N., Walker, G., Jenkins, D., and Smart, W. Where do we go from here? An assessment of navigation performance using a compass versus a GPS unit. Cognition, Technology, \& Work 10 (2008), 231-236. doi:10.1007/ s10111-007-0095-7 